\title{
Role of nuclear-electronic coupling in attosecond photoionization of $\mathbf{H}_{2}$
}

\author{
Anna L. Wang $\odot,{ }^{1,2, *}$ Vladislav V. Serov $\odot,{ }^{3}$ Andrei Kamalov, ${ }^{4,1}$ Philip H. Bucksbaum, ${ }^{2,1,5}$ \\ Anatoli Kheifets, ${ }^{6}$ and James P. Cryan $\oplus^{4,1, \dagger}$ \\ ${ }^{1}$ Stanford PULSE Institute, SLAC National Accelerator Laboratory, Menlo Park, California 94025, USA \\ ${ }^{2}$ Department of Applied Physics, Stanford University, Stanford, California 94305, USA \\ ${ }^{3}$ Department of Theoretical Physics, Saratov State University, 83 Astrakhanskaya, Saratov 410012, Russia \\ ${ }^{4}$ Linac Coherent Light Source, SLAC National Accelerator Laboratory, Menlo Park, California 94025, USA \\ ${ }^{5}$ Department of Physics, Stanford University, Stanford, California 94305, USA \\ ${ }^{6}$ Research School of Physics, Australian National University, Canberra ACT 2601, Australia
}

(Received 22 June 2021; revised 30 September 2021; accepted 3 December 2021; published 27 December 2021)

\begin{abstract}
The separation of electronic and nuclear dynamics due to differing timescales is a useful concept for understanding ground-state molecular systems. However, coupling between these degrees of freedom is critical to understanding the evolution of most excited-state systems. We measure two-photon ionization delays of $\mathrm{H}_{2}$ and compare to calculations of the same measurement in a frozen-nuclei approximation. We find discrepancies between the vibrationally resolved measurement and bond-length-dependent theory, suggesting that nuclear motion affects $\mathrm{H}_{2}$ photoionization on attosecond timescales. We ascribe our observation to nuclear-electronic channel coupling between continuum vibrational states. Our results demonstrate that nuclear-electronic coupling cannot be neglected in the sudden ionization of molecules containing light atoms.
\end{abstract}

DOI: 10.1103/PhysRevA.104.063119

\section{INTRODUCTION}

The coupling of electronic and nuclear motion is a fundamental problem in photochemistry. This coupling is most prominent when the electronic and vibrational energy scales are similar. One situation in which this can occur is in molecular systems containing light, fast-moving nuclei, such as hydrogen, which is an important component of light-sensitive biomolecules. We investigate how electron dynamics are influenced by nuclear motion in hydrogen-containing systems, on their natural timescale of attoseconds. To do this, we measure the attosecond photoionization time delays of molecular hydrogen $\left(\mathrm{H}_{2}\right)$, which is the simplest system that can display nuclear-electronic coupling.

The photoionization time delay is a differential measurement of the phase of the two-photon ionization matrix element, and can reveal ionization dynamics when compared to a set of models. Correlated electronic phenomena such as autoionization resonances [1-5], continuum channel coupling [6-8], and shake-up ionization [9] have been studied in atomic systems using photoionization time delays. Molecular systems provide even more opportunities to study electron dynamics in photoionization [10-14]. Depending on the

\footnotetext{
*annaliw@stanford.edu
}

$\dagger$ jcryan@slac.stanford.edu

Published by the American Physical Society under the terms of the Creative Commons Attribution 4.0 International license. Further distribution of this work must maintain attribution to the author(s) and the published article's title, journal citation, and DOI. geometry and number of nuclei, photoionization from molecules may be affected by multiple scattering, coupling between multiple ionization channels, and interaction with the nuclear degrees of freedom. The photoionization delay is particularly sensitive to molecular shape resonances $[10,13,14]$. Moreover, the photoionization delay was also shown to be sensitive to coupling between various ionization channels around resonance features, such as shape resonances, as well as in the vicinity of autoionizing resonances $[14,15]$.

Continuum-channel coupling in molecular photoionization can also occur in a vibrationally excited system where nuclear and electronic timescales match. This is an example of the breakdown of the Born-Oppenheimer, or adiabatic, approximation (BOA), which allows for the separation of nuclear and electronic degrees of freedom in the Hamiltonian. This model will breakdown near conical intersections, where nonadiabatic coupling can cause the exchange of energy between electronic and nuclear degrees of freedom in bound electronic states [16]. In this article we investigate the coupling between continuum states via inelastic electron scattering, which represents a breakdown of the BOA during photoionization. Our work builds on existing knowledge of non-BOA physics in $\mathrm{H}_{2}$ photoionization [17] by revealing continuum channel coupling between discrete $\mathrm{H}_{2}{ }^{+}$vibrational states, with high resolution in both time and energy.

\section{METHODS}

We measure the photoionization delays of molecular hydrogen $\left(\mathrm{H}_{2}\right)$ using a reconstruction of attosecond beating by interfering two-photon transitions (RABBITT) measurement scheme $[18,19]$. RABBITT is a flexible tool for studying 


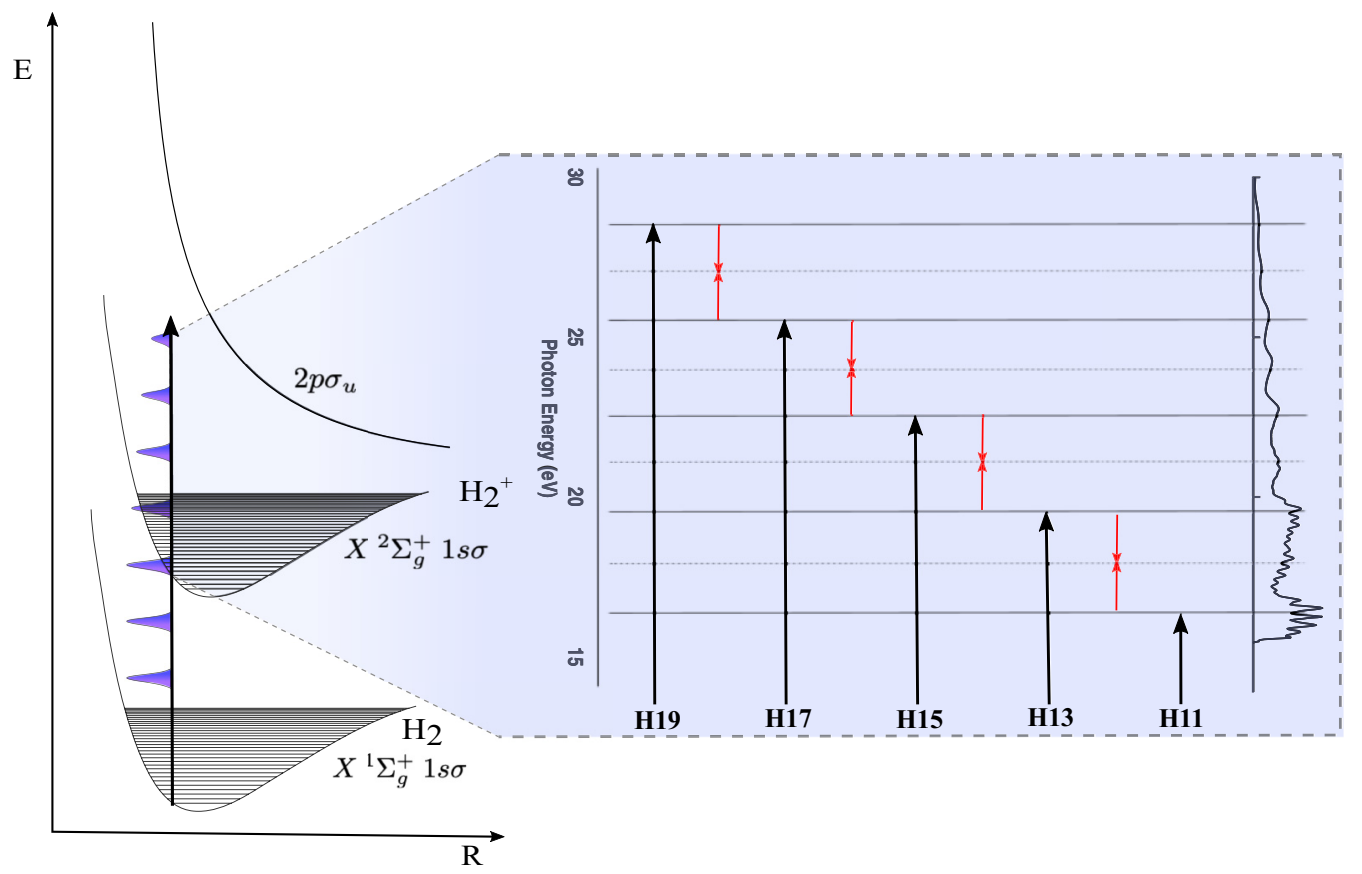

FIG. 1. Schematic for photoionization by a train of attosecond XUV pulses (APT) in the presence of a weak IR field, resulting in our $\mathrm{H}_{2}$ RABBITT measurement. The APT consists of harmonics up to $30 \mathrm{eV}$ (purple), which drives photoionization in $\mathrm{H}_{2}$ molecules for frequencies above the ionization threshold (i.e., harmonics 11-19). The emitted photoelectrons interact with the weak IR field to produce sideband features, illustrated graphically in the inset. The far right-hand side of the inset shows the measured photoelectron spectrum for the combined XUV/IR field with no retardation voltage on the electron spectrometer (see text).

attosecond dynamics in a variety of systems. The technique was originally developed to reconstruct the temporal profile of an attosecond pulse train (APT), and is now frequently used to study photoemission delays [1,3,5-15,17,20-30]. In a RABBITT measurement, we observe modulations in the photoelectron spectrum produced by the combined field of a periodic, extreme ultraviolet (XUV) APT (with temporal spacing $\frac{\pi}{\omega}$ ) and weak infrared (IR) probe field (with central frequency $\omega$ ) as a function of the XUV/IR delay. This produces a photoelectron spectrum with photoemission features from the APT frequency comb and weak sideband features formed by the two-photon (XUV + IR) ionization process. See Fig. 1 for a graphical illustration. As seen in the differential signal displayed in the bottom panel of Fig. 2, the sideband yield oscillates with the XUV/IR delay $\tau$ at twice the probe frequency $(2 \omega)$,

$$
\mathrm{SB}_{2 n}(\tau) \propto \cos \left[2 \omega \tau+\Delta \phi_{2 \omega}(2 n)\right],
$$

where $\Delta \phi_{2 \omega}(2 n)$ is a phase offset in the sideband modulation that varies with sideband order $(2 n)$. This oscillation in the sideband yield is the result of interference between the two different photoionization pathways that produce electrons with the same kinetic energy. In general it is possible to partition $\Delta \phi_{2 \omega}(2 n)$ as follows:

$$
\Delta \phi_{2 \omega}=\Delta \phi_{\mathrm{XUV}}+\Delta \phi_{a},
$$

where $\Delta \phi_{\mathrm{XUV}}$ is a result of the group delay in the APT. The other term, $\Delta \phi_{a}$, is the contribution to $\Delta \phi_{2 \omega}(2 n)$ from the target which encodes photoionization dynamics.

The target-dependent term $\Delta \phi_{a}$ is the result of phase differences in the perturbative, two-photon ionization process that produces the sideband features. This phase difference is frequently decomposed further into a single-photon, XUV ionization contribution $\Delta \phi_{\mathrm{PI}}$ and a measurement-induced contribution, often referred to as the continuum-continuum phase, $\Delta \phi_{\mathrm{CC}}$. This latter approximation is limited to a small set of photoionization cases, and is not valid for low-energy photoelectrons, near continuum resonances, or where multiple ionization states may interact. The separation is further complicated in molecular photoionization, where there may be multiple partial-wave couplings in the continuum [14,31]. In the current study we abstain from partitioning $\Delta \phi_{a}$, because we use a molecular target and part of the measurement takes place near the ionization threshold.

We then use $\Delta \phi_{a}$ to define the two-photon photoionization delay $\tau^{(2)}(E)$,

$$
\tau^{(2)}(E)=\frac{\Delta \phi_{a}}{2 \hbar \omega} \sim \frac{\partial \phi(E)}{\partial E},
$$

since the energy derivative of the spectral phase is the group delay of a wave packet. The conversion to time delay is done to match convention and provide physical interpretation of the phase. $\Delta \phi_{\mathrm{XUV}}$ can similarly be converted to attochirp ( $\left.\tau_{\mathrm{XUV}}\right)$ by dividing by the harmonic spacing. Due to the presence of the IR field, the two-photon time delay $\tau^{(2)}$ is not the same quantity as the electron scattering delay, but it is still a useful observable for studying photoionization dynamics [32]. We will isolate the effects of particular physical properties of $\mathrm{H}_{2}$ photoionization by comparing to a two-color time-dependent Schrödinger equation (TDSE) simulation of the two-photon delay in a model system. 


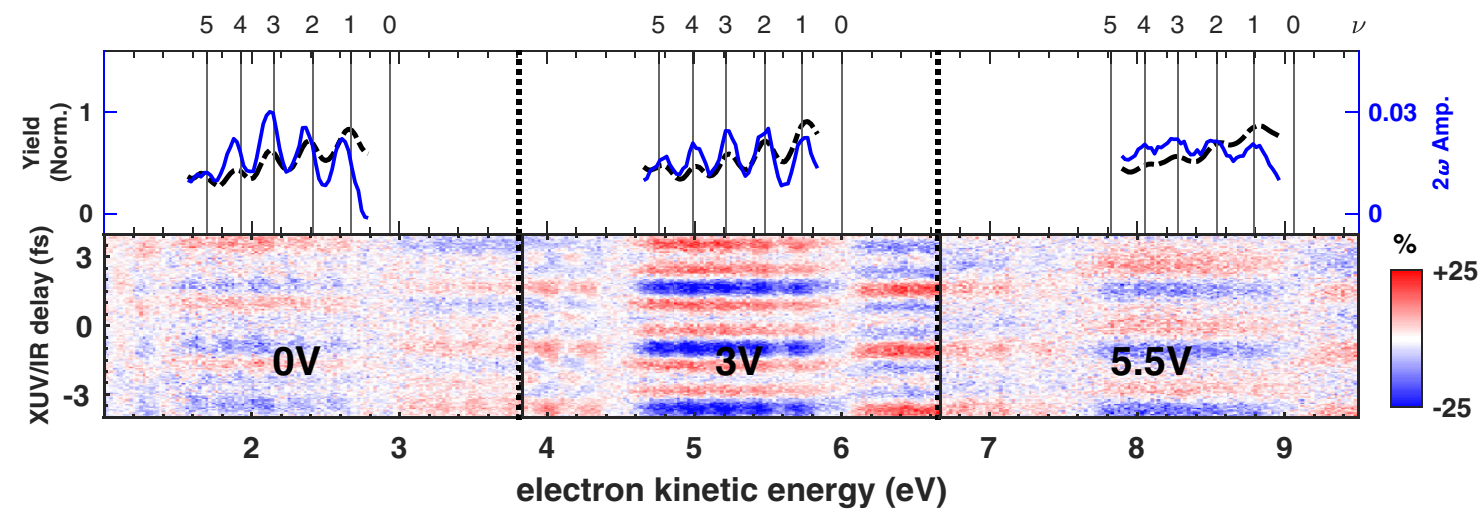

FIG. 2. Two-color photoelectron spectrogram of $\mathrm{H}_{2}$. The bottom panel shows the photoionization spectrum of $\mathrm{H}_{2}$ as a function of XUV/IR time delay, displayed as a percent difference from the time-averaged photoelectron spectrum. The pump/probe oscillations are visible on the time delay axis and vibrational state peaks are visible in the energy axis. A combination of $\omega$ and $2 \omega$ oscillations is visible; the $2 \omega$ oscillations are isolated by Fourier analysis and used for RABBITT analysis (see Supplemental Material [33]). The amplitudes in the top panel demonstrate our vibrational resolution in each sideband. The black dotted curves (left axis) show the normalized, delay-averaged XUV/IR spectrum and the blue curves (right axis) show the Fourier amplitude of the $2 \omega$ oscillations. Each sideband was measured as separate data sets, each with different electron retardation voltages, to optimize spectral resolution (labeled on plot).

\section{A. Measurement}

The XUV APT used to produce the data shown in Fig. 2 is produced by strong-field driven high harmonic generation (HHG). The HHG driving field and RABBITT probe field are both produced by an $810 \mathrm{~nm}, 30 \mathrm{fs}, 100 \mathrm{~Hz}$ repetition rate Ti:sapphire laser. The laser is split into three beams. Two HHG driving beams of about $\sim 14 \mathrm{~mJ}$ pulse energy are temporally overlapped and vertically offset. They are focused together by an $f=5 \mathrm{~m}$ spherical mirror. They cross in an argon gas cell (10 $\mathrm{mm}$ long with 7 Torr pressure) at the laser focus to produce high harmonics. The crossed-beam geometry allows us to separate the XUV radiation from the residual driving field in the far field after the HHG cell and before the RABBITT interaction region. A third, weak $(<3 \mathrm{~mJ})$ probe beam copropagates with the driving field and is temporally advanced with a piezoelectric driven delay stage by $\sim 150$ fs to avoid interference with the HHG process. The delay stage also controls the probe delay in the RABBITT measurement, with $\sim 10$ as step resolution. The probe beam is transmitted through a thin piece of fused silica after the gas cell, which compensates for the $\sim 150$ fs advance introduced upstream and temporally overlaps the IR pulse and APT. At the endstation, the APT and probe are both focused by $\mathrm{a}_{4} \mathrm{C}$ coated focusing optic $(f=10 \mathrm{~cm})$ into the interaction region of a magnetic bottle spectrometer. This focusing optic ultimately defines the bandwidth of the XUV APT by suppressing frequencies above $\sim 30 \mathrm{eV}$, leaving us with harmonics up to H19. For more information on the photon energies and ionization pathways used in this experiment, see Supplemental Material [33]. The $\mathrm{H}_{2}$ and argon samples are introduced into the interaction region by a $35 \mu \mathrm{m}$ gas needle.

The flight tube of the magnetic bottle spectrometer is $1.2 \mathrm{~m}$ long and, as is common to any time-of-flight device, the resolution varies with electron energy. The vibrational states of the hydrogen cation are clearly resolved at low electron energies, and become harder to distinguish above $5 \mathrm{eV}$. We can change which part of the spectrum lies within the high-resolution energy range by applying a retarding voltage to the front of the flight tube. We repeated the RABBITT measurement with each of the sidebands $(12,14$, and 16) in the high-resolution energy region using different retardation potentials $(0,3$, and $5.5 \mathrm{~V}$, respectively), as shown in Fig. 2. Argon provides an energy calibration reference as well as an XUV phase reference for each measurement.

\section{B. Analysis}

To extract the phase $\Delta \phi_{2 \omega}$, we Fourier transform the raw data shown in Fig. 2 along the time delay axis and extract the components with frequency near $2 \omega$. This procedure filters out any contamination of the signal caused by the residual driving laser field, which appears at $1 \omega$, or broadband and dc noise. More details on our Fourier transform analysis can be found in the Supplemental Material [33]. The extracted phase is then corrected for any timing drift in the pump/probe delay. Although the delay stage has high precision, we observe a timing drift over the course of the measurement. We correct this drift by saving the data in chronological bins, each of which contains enough repetitions of the pump/probe delay measurement to give a reasonably accurate estimate of the $(2 \omega)$ RABBITT signal. For each measurement repetition, the time delay values are taken in a random order to further reduce the impact of laboratory-time systematic errors. We then correct the global phase offset of the RABBITT signal of each chronological bin and combine the results to form a single data set. Similarly, the three separate data sets at 0, 3, and $5 \mathrm{~V}$ electron retardation are matched to a global phase offset, given by the phase of the high-energy SB18 electrons. More details on the time drift corrections and global phase corrections can be found in the Supplemental Material [33].

The delay-drift corrected data set has a clear $2 \omega$ oscillation signal at each photoionization peak from which we can extract RABBITT phase values. Our magnetic bottle resolves the vibrational states of $\mathrm{H}_{2}{ }^{+}$in each harmonic band. However, there is still some degree of overlap of adjacent state peaks even with improved resolution after voltage retardation. We take an 
average of the phase inside each peak, using the $2 \omega$ oscillation amplitude to weight each photoelectron energy point. This spectral integration method reduces the impact of the lower amplitude, overlapped parts of the spectrum,

$$
\phi_{\mathrm{SI}}=\arg \left[\sum A_{2 \omega} e^{i \phi_{2 \omega}}\right] .
$$

We use bootstrapping to measure the variation on our phase extraction, repeating the spectral integration analysis on 100 resampled data sets and using the standard error of the extracted phase values to determine the error on our measurement.

The resulting phase extracted from the above analysis is the RABBITT phase $\Delta \phi_{2 \omega}$. To isolate $\Delta \phi_{a}$, we remove $\Delta \phi_{\text {XUV }}$ using a reference measurement [14,27] made in argon. We measure $\Delta \phi^{\mathrm{Ar}}=\Delta \phi_{\mathrm{XUV}}^{\mathrm{Ar}}+\Delta \phi_{\mathrm{CC}}^{\mathrm{Ar}}+\Delta \phi_{\mathrm{PI}}^{\mathrm{Ar}}$, and subtract $\Delta \phi_{\mathrm{CC}}^{\mathrm{Ar}}$ and $\Delta \phi_{\mathrm{PI}}^{\mathrm{Ar}}$ using theoretical values [6,21]. This method requires treating $\Delta \phi_{\mathrm{CC}}^{\mathrm{Ar}}$ and $\Delta \phi_{\mathrm{PI}}^{\mathrm{Ar}}$ as separable at low electron energy, around $2 \mathrm{eV}$, but we find that an enhanced model of the continuum-continuum delays is accurate enough here [21]. The argon reference measurement is taken under the same conditions as $\mathrm{H}_{2}$, so the implicit assumption in this method is that the experiments share the same $\Delta \phi_{\mathrm{XUV}}$ :

$$
\begin{gathered}
\tau_{\mathrm{XUV}}=\tau^{\mathrm{Ar}}-\tau_{\mathrm{PI}}^{\mathrm{Ar}}-\tau_{\mathrm{CC}}^{\mathrm{Ar}}, \\
\tau^{(2)}=\tau^{\mathrm{H}_{2}}-\tau_{\mathrm{XUV}} .
\end{gathered}
$$

\section{Theoretical model and TDSE}

Molecular two-photon ionization phases cannot be accurately calculated through conventional combinations of single-photon ionization models and continuum-continuum phase models [21,31]. This is because molecular systems experience substantial partial wave mixing through two-photon ionization $[14,31]$. We avoid this problem by directly simulating the RABBITT measurement through TDSE calculations of photoionization and photoelectron wave-packet propagation in the combined XUV/IR field.

The simulation XUV APT and weak IR probe are similar to those used in the measurement. Each calculation is integrated over the electron emission direction and averaged over molecular orientation to emulate the magnetic bottle photoelectron spectrometer used in the measurement. TDSE calculations are performed as a function of XUV/IR delays to replicate a RABBITT measurement and extract the $2 \omega$ RABBITT oscillation phase. Numerical details are described in Ref. [34].

One key difference between the measurement and model systems is the treatment of the nuclei. Throughout the calculation, the nuclei are frozen at a single bond length. The RABBITT simulation is repeated for three nuclear separations, which changes the ionization potential and electron kinetic energy in addition to small changes in the scattering potential. The nuclear separations $\left(r_{0}=1.4,1.45,1.5\right.$ a.u. $)$ are close to the equilibrium geometry of $\mathrm{H}_{2}$ and provide a realistic approximation of the molecular scattering potential involved, where the nuclei would only move some very small distance during the photoionization and scattering processes. This reproduces some of the properties of the vibrational spectrum that we observe in the measurement, mainly the asymptotic photoelectron energies for $\mathrm{H}_{2}{ }^{+}$vibrational states $v=1,2,3$. This simplified model lacks the real nuclear dynamics of the system we observe, but it provides a useful comparison for discriminating between significant components of the $\mathrm{H}_{2}$ photoionization Hamiltonian.

\section{RESULTS AND DISCUSSION}

Figure 3 shows both the measured and calculated twophoton ionization delay [Eq. (6)]. We resolve the delay for five vibrational states $(v=1-5)$ of $\mathrm{H}_{2}{ }^{+}$in each sideband (12, 14, 16), shown in black. Figure 3 also shows the results of RABBITT simulations for the three different fixed nuclear separations $R=1.4,1.45,1.5$ a.u. (red markers). Each separate $R$ calculation is connected by a red dashed interpolation to emphasize the differences between different $R$ values.

The main features shown in Fig. 3 are (1) the phase trends between sideband orders and (2) the vibrational state progression within each sideband. The variation of the average experimental delay with sideband order agrees with the general trend of the TDSE calculations. This can be associated with the shape of the cationic potential. The agreement demonstrates that our theory correctly predicts the effect of the cationic potential on two-photon ionization delay, and any further discrepancies are not due to the average molecular potential. In the vibrational progression, the experimental data show a decreasing ionization delay with increasing vibrational state (decreasing electron kinetic energy) in each sideband. In contrast, the TDSE simulations show increasing delay with increasing vibrational state (decreasing electron kinetic energy), which is what we would expect based on the intersideband average trend.

The TDSE calculations reveal the dependence of the photoionization matrix element on internuclear separation. The results for different $R$ values do not lie on a single curve-their potentials are distinct and result in different $\tau^{(2)}(E)$. Other studies of vibrationally resolved photoionization from diatomic molecules have examined this geometry dependence in photoionization delays [13] or a vibrational wave-packet phase $[35,36]$. This ionization delay variation between vibrational states of the cation has been described as non-Franck-Condon physics, where the dipole matrix element varies rapidly with $R$. The variation in curvature of $\tau^{(2)}(E)$ in our TDSE calculations shows the same subtle effect of non-Franck-Condon physics. The vibrational trend of our measurement is beyond the geometric effects that are shown in the calculations.

Instead of simulating vibrational states, the TDSE calculation studies the effect of (static) nuclear geometry on photoionization delay by using frozen nuclei, excluding any vibrational motion effects. Excluding nuclear motion from our model also excludes nuclear-electronic coupling, a possible mechanism for channel coupling between vibrational states in the continuum. In $\mathrm{H}_{2}$ photoionization it is possible to populate multiple fast-moving vibrational states of the cation at nearthreshold electron energies. This allows us to reach conditions where nuclear-electronic coupling occurs because the nuclear and electronic energy scales are similar. There is evidence for nuclear-electronic coupling in this energy range from electron scattering experiments in $\mathrm{H}_{2}[37,38]$ and $\mathrm{H}_{2}{ }^{+}$[39,40], where vibrational state transfer is induced by an energy exchange 

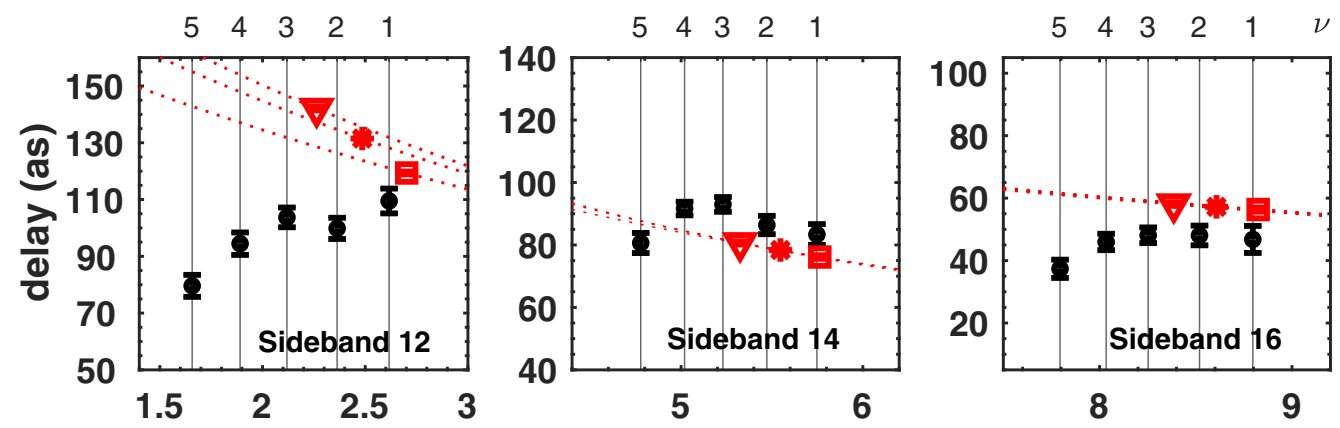

electron kinetic energy $(\mathrm{eV})$

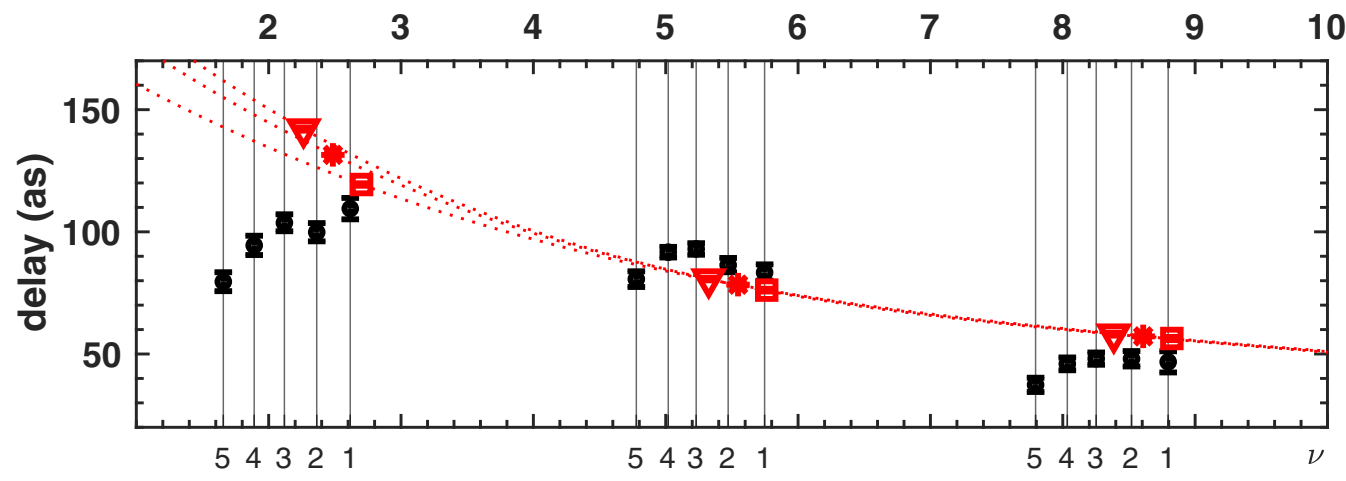

FIG. 3. Two-photon ionization delays for $\mathrm{H}_{2}$. The measured two-photon ionization delay is extracted from Fig. 2 (black circles). This is compared to fixed-nuclei TDSE simulation for three different nuclear separations $R=1.4,1.45,1.5$ a.u. (red triangle, red asterisk, and red square, respectively). Each $R$ simulation is connected by interpolation (red, dashed line). The top panels show a closer view of the bottom panel for each sideband of the RABBITT measurement.

with a free electron. This type of inelastic scattering interaction can be applied to photoionization, often referred to as a half-scattering event, and described as continuum-channel coupling mediated by nuclear-electronic coupling. Attosecond timescale matching between the vibrationally excited nuclei and free electron has also been observed in HHG spectroscopy of $\mathrm{H}_{2}$ and $\mathrm{D}_{2}$ [41].

Our data support nuclear-electronic channel coupling in $\mathrm{H}_{2}$ photoionization. We see the discrepancy between theory and experiment increases with the vibrational state number. This discrepancy represents the BOA nonadiabatic coupling term of the system, which would not be present in the calculation due to the lack of nuclear motion. The nonadiabatic coupling term is proportional to the nuclear momentum, so it should grow with the vibrational state as shown in our data. The effect is also most prominent for near-threshold electron energies in sideband 12, which gives further evidence for non-BOA physics due to energy matching.

\section{CONCLUSION}

We compared measurements of the two-photon ionization delay with fixed-nuclei simulations. Discrepancies between the measured delays and calculated delays increase (1) with an increasing vibrational state within each sideband, which corresponds to an increasing nuclear momentum, and (2) for near-threshold electrons (SB12). Under these two conditions, which correspond to momentum matching between the electrons and nuclei, we may observe nuclear-electronic coupling associated with breakdown of the Born-Oppenheimer approximation. Since nuclear-electronic coupling is the only vibrational state-dependent phenomena that is not accessible in our fixed-nuclei model, it is a likely source of the discrepancy between theory and experiment. Our observations of nuclear-electronic coupling in $\mathrm{H}_{2}$ photoionization motivate further examination of the interaction of hydrogen motion and attosecond electron dynamics in molecular systems.

In the future, we intend to abandon the fixed $R$ approximation and to treat the unrestricted vibrational dynamics. This work implemented within the prolate spheroidal exterior complex scaling method is currently underway. We hope that such an improved theoretical model shall be able to reproduce the experimental data more accurately

\section{ACKNOWLEDGMENTS}

This work was supported by the AMOS program within the Chemical Sciences, Geosciences, and Biosciences Division of the Office of Basic Energy Sciences, Office of Science, U.S. Department of Energy. A.L.W. acknowledges support from the Stanford VPGE EDGE Fellowship. 
[1] V. Gruson, L. Barreau, Á. Jiménez-Galan, F. Risoud, J. Caillat, A. Maquet, B. Carré, F. Lepetit, J.-F. Hergott, T. Ruchon, L. Argenti, R. Taïeb, F. Martín, and P. Salières, Attosecond dynamics through a Fano resonance: Monitoring the birth of a photoelectron, Science 354, 734 (2016).

[2] M. Kotur, D. Guénot, Á. Jiménez-Galán, D. Kroon, E. W. Larsen, M. Louisy, S. Bengtsson, M. Miranda, J. Mauritsson, C. L. Arnold, S. E. Canton, M. Gisselbrecht, T. Carette, J. M. Dahlström, E. Lindroth, A. Maquet, L. Argenti, F. Martín, and A. L'Huillier, Spectral phase measurement of a Fano resonance using tunable attosecond pulses, Nat. Commun. 7, 10566 (2016).

[3] C. Cirelli, C. Marante, S. Heuser, C. L. M. Petersson, Á. Jiménez-Galán, L. Argenti, S. Zhong, D. Busto, M. Isinger, S. Nandi, S. Maclot, L. Rading, P. Johnsson, M. Gisselbrecht, M. Lucchini, L. Gallmann, J. M. Dahlström, E. Lindroth, A. L'Huillier, F. Martín, and U. Keller, Anisotropic photoemission time delays close to a Fano resonance, Nat. Commun. 9, 955 (2018).

[4] D. Busto, L. Barreau, M. Isinger, M. Turconi, C. Alexandridi, A. Harth, S. Zhong, R. J. Squibb, D. Kroon, S. Plogmaker, M. Miranda, Á. Jiménez-Galán, L. Argenti, C. L. Arnold, R. Feifel, F. Martín, M. Gisselbrecht, A. L'Huillier, and P. Salières, Timefrequency representation of autoionization dynamics in helium, J. Phys. B: At., Mol. Opt. Phys. 51, 044002 (2018).

[5] L. Barreau, C. L. M. Petersson, M. Klinker, A. Camper, C. Marante, T. Gorman, D. Kiesewetter, L. Argenti, P. Agostini, J. González-Vázquez, P. Salières, L. F. DiMauro, and F. Martín, Disentangling Spectral Phases of Interfering Autoionizing States from Attosecond Interferometric Measurements, Phys. Rev. Lett. 122, 253203 (2019).

[6] S. Saha, J. Jose, P. C. Deshmukh, G. Aravind, V. K. Dolmatov, A. S. Kheifets, and S. T. Manson, Wigner time delay in photodetachment, Phys. Rev. A 99, 043407 (2019).

[7] S. Banerjee, P. C. Deshmukh, A. S. Kheifets, and S. T. Manson, Effects of spin-orbit-interaction-activated interchannel coupling on photoemission time delay, Phys. Rev. A 101, 043411 (2020).

[8] I. Jordan, M. Huppert, S. Pabst, A. S. Kheifets, D. Baykusheva, and H. J. Wörner, Spin-orbit delays in photoemission, Phys. Rev. A 95, 013404 (2017).

[9] M. Ossiander, F. Siegrist, V. Shirvanyan, R. Pazourek, A. Sommer, T. Latka, A. Guggenmos, S. Nagele, J. Feist, J. Burgdörfer, R. Kienberger, and M. Schultze, Attosecond correlation dynamics, Nat. Phys. 13, 280 (2017).

[10] M. Huppert, I. Jordan, D. Baykusheva, A. von Conta, and H. J. Wörner, Attosecond Delays in Molecular Photoionization, Phys. Rev. Lett. 117, 093001 (2016).

[11] S. Haessler, B. Fabre, J. Higuet, J. Caillat, T. Ruchon, P. Breger, B. Carré, E. Constant, A. Maquet, E. Mével, P. Salières, R. Taïeb, and Y. Mairesse, Phase-resolved attosecond nearthreshold photoionization of molecular nitrogen, Phys. Rev. A 80, 011404(R) (2009).

[12] J. Vos, L. Cattaneo, S. Patchkovskii, T. Zimmermann, C. Cirelli, M. Lucchini, A. Kheifets, A. S. Landsman, and U. Keller, Orientation-dependent stereo Wigner time delay and electron localization in a small molecule, Science 360, 1326 (2018).

[13] S. Nandi, E. Plésiat, S. Zhong, A. Palacios, D. Busto, M. Isinger, L. Neoričić, C. L. Arnold, R. J. Squibb, R. Feifel,
P. Decleva, A. L'Huillier, F. Martín, and M. Gisselbrecht, Attosecond timing of electron emission from a molecular shape resonance, Sci. Adv. 6, eaba7762 (2020).

[14] A. Kamalov, A. L. Wang, P. H. Bucksbaum, D. J. Haxton, and J. P. Cryan, Electron correlation effects in attosecond photoionization of $\mathrm{CO}_{2}$, Phys. Rev. A 102, 023118 (2020).

[15] S. Beaulieu, A. Comby, A. Clergerie, J. Caillat, D. Descamps, N. Dudovich, B. Fabre, R. Géneaux, F. Légaré, S. Petit, B. Pons, G. Porat, T. Ruchon, R. Taïeb, V. Blanchet, and Y. Mairesse, Attosecond-resolved photoionization of chiral molecules, Science 358, 1288 (2017).

[16] G. A. Worth and L. S. Cederbaum, Beyond Born-Oppenheimer: Molecular dynamics through a conical intersection, Annu. Rev. Phys. Chem. 55, 127 (2004).

[17] L. Cattaneo, J. Vos, R. Y. Bello, A. Palacios, S. Heuser, L. Pedrelli, M. Lucchini, C. Cirelli, F. Martín, and U. Keller, Attosecond coupled electron and nuclear dynamics in dissociative ionization of $\mathrm{H}_{2}$, Nat. Phys. 14, 733 (2018).

[18] P. M. Paul, E. S. Toma, P. Breger, G. Mullot, F. Augé, P. Balcou, H. G. Muller, and P. Agostini, Observation of a train of attosecond pulses from high harmonic generation, Science 292, 1689 (2001).

[19] H. G. Muller, Reconstruction of attosecond harmonic beating by interference of two-photon transitions, Appl. Phys. B 74, s17 (2002).

[20] C. Alexandridi, D. Platzer, L. Barreau, D. Busto, S. Zhong, M. Turconi, L. Neoričić, H. Laurell, C. L. Arnold, A. Borot, J.-F. Hergott, O. Tcherbakoff, M. Lejman, M. Gisselbrecht, E. Lindroth, A. L'Huillier, J. M. Dahlström, and P. Salières, Attosecond photoionization dynamics in the vicinity of the Cooper minima in argon, Phys. Rev. Research 3, L012012 (2021).

[21] J. M. Dahlström, A. L'Huillier, and A. Maquet, Introduction to attosecond delays in photoionization, J. Phys. B: At., Mol. Opt. Phys. 45, 183001 (2012).

[22] R. Pazourek, S. Nagele, and J. Burgdörfer, Attosecond chronoscopy of photoemission, Rev. Mod. Phys. 87, 765 (2015).

[23] M. Schultze, M. Fieß, N. Karpowicz, J. Gagnon, M. Korbman, M. Hofstetter, S. Neppl, A. L. Cavalieri, Y. Komninos, T. Mercouris, C. A. Nicolaides, R. Pazourek, S. Nagele, J. Feist, J. Burgdörfer, A. M. Azzeer, R. Ernstorfer, R. Kienberger, U. Kleineberg, E. Goulielmakis, F. Krausz, and V. S. Yakovlev, Delay in photoemission, Science 328, 1658 (2010).

[24] K. Klünder, J. M. Dahlström, M. Gisselbrecht, T. Fordell, M. Swoboda, D. Guénot, P. Johnsson, J. Caillat, J. Mauritsson, A. Maquet, R. Taïeb, and A. L'Huillier, Probing single-photon ionization on the attosecond time scale, Phys. Rev. Lett. 106, 143002 (2011).

[25] D. Guénot, K. Klünder, C. L. Arnold, D. Kroon, J. M. Dahlström, M. Miranda, T. Fordell, M. Gisselbrecht, P. Johnsson, J. Mauritsson, E. Lindroth, A. Maquet, R. Taïeb, A. L'Huillier, and A. S. Kheifets, Photoemission-time-delay measurements and calculations close to the $3 s$-ionizationcross-section minimum in Ar, Phys. Rev. A 85, 053424 (2012).

[26] A. S. Kheifets, Time delay in valence-shell photoionization of noble-gas atoms, Phys. Rev. A 87, 063404 (2013).

[27] D. Guénot, D. Kroon, E. Balogh, E. W. Larsen, M. Kotur, M. Miranda, T. Fordell, P. Johnsson, J. Mauritsson, M. 
Gisselbrecht, K. Varjù, C. L. Arnold, T. Carette, A. S. Kheifets, E. Lindroth, A. L'Huillier, and J. M. Dahlström, Measurements of relative photoemission time delays in noble gas atoms, J. Phys. B: At., Mol. Opt. Phys. 47, 245602 (2014).

[28] C. Palatchi, J. M. Dahlström, A. S. Kheifets, I. A. Ivanov, D. M. Canaday, P. Agostini, and L. F. DiMauro, Atomic delay in helium, neon, argon and krypton, J. Phys. B: At., Mol. Opt. Phys. 47, 245003 (2014).

[29] M. Sabbar, S. Heuser, R. Boge, M. Lucchini, T. Carette, E. Lindroth, L. Gallmann, C. Cirelli, and U. Keller, Resonance Effects in Photoemission Time Delays, Phys. Rev. Lett. 115, 133001 (2015).

[30] D. Kiesewetter, R. R. Jones, A. Camper, S. B. Schoun, P. Agostini, and L. F. DiMauro, Probing electronic binding potentials with attosecond photoelectron wavepackets, Nat. Phys. 14, 68 (2017).

[31] D. Baykusheva and H. J. Wörner, Theory of attosecond delays in molecular photoionization, J. Chem. Phys. 146, 124306 (2017).

[32] L. Argenti, A. Jiménez-Galán, J. Caillat, R. Taïeb, A. Maquet, and F. Martín, Control of photoemission delay in resonant two-photon transitions, Phys. Rev. A 95, 043426 (2017).

[33] See Supplemental Material at http://link.aps.org/supplemental/ 10.1103/PhysRevA.104.063119 for further information on experimental apparatus, details of data analysis, and raw photoelectron spectra.
[34] V. V. Serov and A. S. Kheifets, Time delay in XUV/IR photoionization of $\mathrm{H}_{2} \mathrm{O}$, J. Chem. Phys. 147, 204303 (2017).

[35] Y. Nabekawa, Y. Furukawa, T. Okino, A. Amani Eilanlou, E. J. Takahashi, K. Yamanouchi, and K. Midorikawa, Settling time of a vibrational wavepacket in ionization, Nat. Commun. 6, 8197 (2015).

[36] T. Nishi, E. Lötstedt, and K. Yamanouchi, Time delay in the coherent vibrational motion of $\mathrm{H}_{2}{ }^{+}$created by ionization of $\mathrm{H}_{2}$, Phys. Rev. A 102, 051101(R) (2020).

[37] G. J. Schulz, Vibrational excitation of $\mathrm{N}_{2}, \mathrm{CO}$, and $\mathrm{H}_{2}$ by electron impact, Phys. Rev. 135, A988 (1964).

[38] P. D. Burrow and G. J. Schulz, Vibrational excitation by electron impact near threshold in $\mathrm{H}_{2}, \mathrm{D}_{2}, \mathrm{~N}_{2}$, and CO, Phys. Rev. 187, 97 (1969).

[39] M. Fifirig and M. Stroe, Dissociation of $\mathrm{H}_{2}{ }^{+}$ions by collisions with electrons, J. Phys. B: At., Mol. Opt. Phys. 44, 085202 (2011).

[40] S. Krohn, H. Kreckel, L. Lammich, M. Lange, J. Levin, D. Schwalm, D. Strasser, R. Wester, A. Wolf, and D. Zajfman, Electron induced vibrational deexcitation of the molecular ions $\mathrm{H}_{2}{ }^{+}$and $\mathrm{D}_{2}{ }^{+}$, in Dissociative Recombination of Molecular Ions with Electrons, edited by S. L. Guberman (Springer, Boston, MA, 2003), pp. 127-138.

[41] S. Baker, J. S. Robinson, C. A. Haworth, H. Teng, R. A. Smith, C. C. Chirilă, M. Lein, J. W. G. Tisch, and J. P. Marangos, Probing proton dynamics in molecules on an attosecond time scale, Science 312, 424 (2006). 